\title{
El debate sobre las desigualdades contemporáneas: ¿puede excluirse la exclusión social?
}

\author{
The Debate on the Contemporary Inequalities: Social exclusion can \\ be excluded?
}

\section{Marcelo Arnold Cathalifaud}

Departamento de Antropología, Facultad de Ciencias Sociales, Universidad de Chile

\section{Resumen}

\begin{abstract}
El presente artículo examina el escenario actual de la crisis mundial. Concentra nuestra atención la modalidad de organización de la sociedad contemporánea que, admite desigualdades sociales de todo tipo, mientras determina las reacciones frente dicha situación. Se propone moderar el optimismo de las interpretaciones sobre las desigualdades -indicadas desde el lado de la exclusión social-, especialmente cuando se las señala como anomalías corregibles con decisiones o instructivos morales; también cuando se descuida su carácter dinámico y multidimensional, reduciéndolas a temas de pobreza o de distribución de riqueza. Se concluye que las desigualdades se han multiplicado y están determinadas por las formas que han asumido las estructuras de la sociedad; al mismo tiempo, explicamos cómo globalmente, o desde sus distintos sistemas, esas desigualdades no son aceptables y se anticipa que aumentaran las demandas hacia el sistema político exigiendo su superación o contención.
\end{abstract}

Palabras Clave: Sociedad Contemporánea; Diferenciación Funcional; Integración Social; Desigualdades Sociales; Inclusión/ Exclusión

\begin{abstract}
This article examines the current scenario of the global crisis. We focus our attention on the mode of organization of contemporary society, which admits inequalities of all kinds, while determines the reactions to this situation. We intend to temper the optimism of the interpretations of inequalities - listed from the side of social exclusion- especially when they are pointed out as anomalies susceptible of being corrected with decisions or moral instructions; also when their dynamical and multidimensional character, are reduced to poverty or wealth distribution issues. It is concluded that inequalities have multiplied and are determined by the forms that the structures of society have taken on; at the same time, explain how globally, or from their different systems, these inequalities are not acceptable and it is anticipated that their demands to political system will increase, requiring it's overcoming or contention.
\end{abstract}

Keywords: Contemporary Society; Functional Differentiation; Social Integration; Social Inequalities; Inclusion/ Exclusion

\section{Introducción: La conflictividad mundial}

Ante el actual volumen de la población mundial, que es indicativo de la prosperidad y el mejoramiento sostenido de las condiciones de vida contemporáneas, sorprende el hecho de que la sociedad se observe como un surtidor de desigualdades sociales, y que en un planeta cada vez más integrado y globalizado, su nivel de cohesión es cuestionado por un 
generalizado descontento social. Durante el año pasado (2011) las protestas sociales se multiplicaron en Latinoamérica, en los Estados Unidos, en países europeos e incluso en aquellos estados donde los medios de control y represión han sido tradicionalmente efectivos. Aumentó significativamente la cantidad de personas y grupos que expresan la frustración de sus expectativas y reclaman por sus derechos. Las manifestaciones se han hecho más frecuentes, conflictivas y acumulan más resentimiento.

El malestar generalizado, la composición social de los manifestantes, el uso intensivo de las redes sociales para potenciar sus movilizaciones, los cada vez más altos niveles de rechazo público a la corrupción y el abuso de poder, configuran como la sorpresa del siglo veintiuno una rebelión mundializada. La agitación se vincula con un rápido aumento de la decepción frente a la falta de previsión del futuro y ante las inequidades atribuidas a los programas económicos que impulsan el crecimiento mundial, como también frente a la ineficacia de las instituciones políticas para controlar actividades que, como se denuncia, operan a gran escala y con una enorme autonomía en perjuicio de las mayorías.

Para comprender las actuales desigualdades sociales, y los niveles de las protestas que generan sus efectos, debe considerarse la globalización de las formas capitalistas y de las empresas transnacionales, la normalización de las comunicaciones digitalizadas, la formación de audiencias globales, la mundialización de los derechos humanos y la asimilación de los valores democráticos y de justicia social en la mayoría de las regiones del planeta. Estas condiciones, que se refuerzan mutuamente, reflejan una sociedad cuya evolución depende de la variación y recombinación de componentes y procesos a escala planetaria. Chile en su posbicentenario no es ajeno a esta situación y, a nivel local, representa una muestra de problemas que tienen fundamentos y expresiones equivalentes en ciudades como Madrid, Bogotá, Beijing, Zengcheng, Moscú, Londres, Nueva York, Tel Aviv, Montreal o Ciudad de México.

En lo que sigue observaremos, desde una perspectiva sistémica (Arnold 2008a), los fundamentos estructurales de las desigualdades que darían origen a las protestas sociales en relación con los cambios en los modos de integración social de los individuos a la sociedad (Lockwood 1964); luego trataremos los desafíos que enfrenta el sistema político que debe procesar, en primera línea, las deslegitimaciones y manifestaciones más agudas ante las desigualdades sociales. Finalizamos con algunas reflexiones sobre las posibilidades de la inclusión en la sociedad funcionalmente diferenciada. La pregunta que nos guía es si acaso la sociedad contemporánea, en razón a su conformación, cuenta con las condiciones para eliminar de su operar normal las exclusiones sociales.

\section{Las estructuras que incluyen... también excluyen}

Las formas de integración social dependen de las características de la sociedad en sus distintas dimensiones, niveles, momentos y regiones. Si se modifican sus estructuras, como ha ocurrido, se anticipan consecuencias. Estas últimas se vinculan con el explosivo desencadenamiento de las protestas sociales globales ante las crecientes desigualdades sociales.

La progresión de manifestaciones ha dejado al descubierto el descontento de gran parte de la población mundial y la dificultad para contener las tensiones sociales. Se confirma la pérdida de la racionalidad global en la sociedad, y la ausencia de un primado ordenador o coordinador central para sus operaciones, sea éste la religión, la moral, la política, la ciencia, la economía u otro.

La forma predominante de la sociedad se califica como funcionalmente diferenciada. Como la describe Luhmann (e.o. 1998), alude a que sus componentes emergen desde selectividades que se reproducen valiéndose de la distinción entre sistema y entorno, y de las posibilidades de heterorreferencia y autorreferencia que se autoproporcionan. Así, por 
ejemplo, las leyes determinan que no puede hacerse justicia por cuenta propia, y la religión prescribe que no se puede adorar a Dios de cualquier manera. Estas diferenciaciones provocan un acelerado dinamismo en la sociedad, ya que ninguna relación queda fija y todo lo probable se hace contingente. Se incrementan operaciones paradójicas o contradictorias que, aunque parecen cognitivamente inescrutables 0 irracionales, son admisibles en su reproducción, pues son lados, aunque opuestos, de una misma forma. Paralelamente, las imputaciones de causas y efectos se desplazan a través de sistemas autónomos, impidiendo observaciones unitarias que permitan anticipar conexiones o interrelaciones. Es por ello que el conocimiento de la sociedad exige de la mejor ciencia disponible.

En forma gradual la sociedad ha ido componiéndose de sistemas autonomizados. Estos construyen y resguardan sus límites estructurándose bajo modos especializados de funcionamiento, estabilizando provisionalmente sus condiciones con reglas específicas de operación. De este modo la justicia, y la noción de lo justo, pasan a ser exclusividad del derecho, que traduce lo justo como lo legal; la verdad y sus criterios de objetividad, a ser tratados como asuntos de la ciencia; la belleza, determinada por los cánones del arte y los expertos en moda, y así en los otros casos. Por eso las cosas se ven mal cuando el deporte, que tiene una función específica, pasa a ser tema de negocios o cuando los matrimonios, que deben basarse en el amor, se deciden por conveniencia familiar.

La modalidad organizativa de la sociedad se acompaña -más bien se resguarda- con un incremento en la comunicación de conflictos. Estos se hacen patentes, por ejemplo, cuando las sentencias legales se cuestionan desde el punto de vista de la ética, o las ganancias económicas se confrontan con los derechos a la salud; más cuando las sentencias son legales, a no ser que las leyes, con leyes, se modifiquen -con prescindencia de objeciones éticas-, y las ganancias sigan requiriéndose dentro de la esfera económica, con omisión de sus repercusiones en otros ámbitos. La multiplicación de estas situaciones aumenta la irritabilidad social.

\section{El paso del ser humano a la "forma" persona}

La aceleración de los procesos de diferenciación, que también marca la crisis de representación y el imperio de la incertidumbre en la sociedad, se relaciona con cambios radicales en los modos con que los seres humanos, como totalidades biopsíquicas, se vinculan con la sociedad.

Los sistemas sociales, que presentan distintos niveles en sus equipamientos, incrementan la complejidad de la sociedad y, a la vez, generan horizontes específicos de posibilidades y restricciones para la integración de los individuos. Estos quedan en manos de operaciones 'ciegas' que son indiferentes a sus motivos y necesidades, es decir, no consideran nada más allá de las premisas con las cuales se regulan. De esta forma, la sociedad dispone en su estructura de mecanismos que la abastecen ininterrumpidamente de conflictos, demandas, exigencias, oportunidades y derechos, que al replicarse multiplican los problemas que afectan a los individuos y sus expectativas. Su integración se transforma en un desafío ante las limitaciones que ofrecen, con sus formas específicas, los componentes sistémicos de la sociedad, los que, a su vez, están imposibilitados de tratarlos en su totalidad, a pesar de que, para poder ser considerados, requieran ser cada vez más individualizados (Mascareño 2008).

En el camino, se ha hecho admisible la separación entre el 'sí mismo' y el desempeño de roles. La identidad se transforma en problemática abriéndose a numerosos planos, al punto que los individuos solo pueden considerarse socialmente bajo personificaciones específicas, por ejemplo, como artistas, pacientes, manifestantes, académicos, trabajadores, consumidores, estudiantes, víctimas, esposas, auditores, fieles, electores, etcétera. Todo pasa por el reconocimiento de los seres humanos como individuos 
personalizables, es decir, sujetos disponibles para una amplia y segmentada gama de expectativas. Estas formas, "las personas" (Luhmann 1991), que representan una limitación de las posibilidades individuales, son referencias indispensables para las nuevas estructuras sociales.

La condición policontextual alcanzada por la sociedad permite que los procesos de integración social se efectúen por medio de mecanismos específicos de inclusión/ exclusión (Luhmann 1995). Este código, que se sobrepone a todos los sistemas sociales (Stichweh 1997), produce un dinámico, expansivo e inevitable espectro de desigualdades, pues con ellos los sistemas fijan formas particulares de integración social, cuyos resultados se proyectan en diferencias en salarios, educación, vivienda, justicia o atención de salud; también según orientación sexual, condición étnica, edad, apariencia física, acceso a Internet, participación política, acceso a seguridad, exposición a contaminación, acceso a transporte, etcétera. La popularización del concepto de exclusión se conecta con los aspectos más conflictivos de estos nuevos niveles de complejidad social, cuya atención, comprensión e intervención implica abordar lo diverso, dinámico y relacional de tales procesos (e.o. Mathieson et al. 2008).

Dada su complejidad, la sociedad puede producir, sin colapsar, desigualdades de todos los tipos y grados. Aunque se multipliquen las exclusiones, se inhibe que puedan ser totales; quienes están en sus extremos son acogidos por organizaciones de asistencia social o caridad. Incluso pueden observarse mixturas de "exclusiones en la inclusión" o "inclusiones en la exclusión" (Robles 2000) -según como se mire, por ejemplo, el caso de los trabajadores ilegales. Por otra parte, las vinculaciones con un sistema social, o con un par de ellos, no se corresponden con una integración plena. Se es paciente en la salud y no en el arte; se puede ser posgraduado, pero también desempleado; se puede habitar en un condominio de clase alta, pero vivir inseguro. Los individuos pueden estar incluidos parcialmente $y$, a la vez, parcialmente excluidos, o a lo largo de sus vidas experimentar multiinclusiones y multiexclusiones.

Tampoco se trata de incluirse de cualquier forma. Puede cuestionarse la suposición de que la integración social sea siempre positiva, pues, como se sabe, los deudores deben estar incluidos en el sistema económico y los delincuentes en el jurídico. Así, la exclusión puede servir como recurso, en contra de las expectativas sociales, para sustraerse de estar incluido postergando el formalizar relaciones de pareja y con ello sus compromisos, para evitar la atención de salud y optar por la eutanasia, rechazar una cuenta en Facebook para conservar la privacidad o abstenerse de votar para oponerse a los acuerdos políticos.

\section{Sobre los determinismos estructurales y sus conflictos}

La diferenciación funcional diversifica y amplifica las desigualdades sociales -indicadas desde el lado de la exclusión social-, proporcionando causas para los movimientos de protesta. La frase símbolo 'somos el 99\%' testimonia que prácticamente nadie, de acuerdo con sus expectativas, cree estar plenamente incluido en la sociedad contemporánea. Incluso en Nueva York, los manifestantes exigen la plena inclusión y rechazan que sus barreras queden para beneficios de los pocos que acumulan los rendimientos de los muchos. Si bien los movilizados no pueden considerar todas las posibilidades que fundamentan sus motivos, ya que son prisioneros de sus propios puntos ciegos, con sus manifestaciones cuestionan la naturalidad del orden social, declaran inconveniente el sometimiento a sus instituciones custodias y advierten sobre los inminentes peligros del descontrol planetario. Su función es insustituible.

En principio, los motivos de las protestas, y los movimientos sociales que las proyectan, son congruentes con las nuevas estructuras de la sociedad, para las cuales el origen social (o el género o la condición étnica) no es un motivo generalizado de exclusión. Más bien todos deben estar en condiciones de ser excluidos... o incluidos. Justamente, por eso, los 
movimientos sociales se oponen fuertemente a que los intereses económicos, por ejemplo, interpongan sus criterios en ámbitos como la política, la educación o la salud, y tratan esas interferencias como corrupción. Así, en formato más acotado, se rechazan las diferencias por apariencia física para ser atendidos o postular a un empleo, las que se catalogan como discriminaciones.

Se ha configurado el ideal de que todos debemos ser tratados como iguales, no solo por justicia, sino que por ser lo más adecuado para el funcionamiento de la sociedad. Desde esa premisa, la integración social debe basarse en que todos puedan acceder, en las mismas condiciones, a todos los sistemas funcionales, donde nadie pueda arrogarse privilegios frente a leyes que deben aplicarse a todos; por ejemplo al trabajo, cuyo acceso debe basarse en méritos y remunerarse como corresponde; o a los derechos políticos, que deben ser válidos para todos los ciudadanos; o a la educación formal, entendida como parte de los derechos humanos; o a la religión, por cuanto todos pueden creer o no en dioses, o a la economía, donde no puede negarse la venta a quien pueda pagar.

Si bien la diferenciación funcional no elimina entre sus efectos la riqueza $u$ otras inequidades que rebotan en otros sistemas, esas diferencias, en cuanto producen jerarquías permanentes, se interponen a las condiciones de funcionamiento de la sociedad. Por eso, aunque los individuos, en casi todos los sentidos, sean diferentes, deben igualarse. En la sociedad funcionalmente diferenciada todos, a priori, son iguales en su potencialidad de inclusión. Los procesos de ciudadanización o universalización de los derechos cívicos, la democratización o universalización de las capacidades de decisión política, los impuestos y el control de las herencias como medios redistributivos de la riqueza, la educación pública gratuita, o la salud, vivienda, trabajo y ocio, concebidos como derechos, junto con la burocracia pública y privada, son mecanismos disponibles para tal objetivo. Sin embargo, como estos dispositivos no operan como se espera y tampoco están presentes de la misma forma en todas las regiones, hacen que en la sociedad contemporánea se combinen, explosivamente, las nuevas formas organizativas con una "estructura de clases sociales" (Luhmann 1985) y con los espacios familísticos del orden segmentario precedente.

Las fricciones entre las distintas modalidades de estructuración social producen los mayores descontentos, sobre todo entre aquellas comunidades que quieren salir o nunca regresar al viejo orden. Los estratos medios emergentes, en términos sociales, los jóvenes, en términos generacionales, y Latinoamérica, en términos regionales, constituyen ejemplos agudos de estas demandas. En esos ámbitos, la magnitud de las exclusiones es equivalente a las demandas por la mayor inclusión.

Los requerimientos tradicionales para la integración social en sociedades estratificadas o segmentarias no son aceptables, su menoscabo sustenta las protestas más masivas y globales. Estas exigen concretar las posibilidades que ofrece la nueva forma de diferenciación de la sociedad. A nivel local, un ex candidato a la presidencia, interpretando el malestar, señalaba: "el Chile segmentado, clasista, de castas, oligárquico y racista no resiste el peso de la historia"1. Ello informa (a nombre de la 'historia') que las normalidades de ayer hoy son injustas y supone, correctamente, que las exclusiones vinculadas a condiciones de origen familiar, de clase social, étnica o de género son desviaciones o defectos que deben eliminarse. Por eso, y con la mayor indignación, se denuncian las exclusiones que reproducen 'injustamente' las desigualdades sociales.

Observando las trasgresiones al modelo de la igualdad, podemos interpretar mejor la fuerza de las demandas asociadas al ámbito de la educación formal, y no extrañarnos ante la adhesión que concitan los movimientos estudiantiles. Como se sabe, el paso por el sistema educacional, que concentra las mejores oportunidades -opciones de

\footnotetext{
${ }^{1}$ Columna Diario El Mercurio, viernes 10 de agosto de 2012, A2.
} 
"convertibilidad" (Cadenas 2012)- para la integración social, supone la aplicación de criterios fundados en méritos, cuyo efecto es redistribuir posiciones. La paradoja es que la educación distribuye posiciones desiguales, la evidencia empírica sobre esta función es abrumadora. Dados sus procesos selectivos, y no obstante los esfuerzos por contrarrestar este funcionamiento, parece tener a la desigualdad como inevitable resultado. Más aún, la búsqueda de 'ascenso' o movilidad social, a través de sus certificaciones, coloca bajo observación el dilema entre perseguir la igualdad de oportunidades o la igualdad en los resultados. En el momento de acceso la desigualdad educativa es injusta, cuando no ilegítima; con respecto a las ventajas que finalmente produce el rendimiento educacional, el debate es más abierto (¿deben algunas profesiones ser mejor remuneradas que otras?, ¿cuánto más pueden serlo?, ¿cómo reconocer los talentos o el esfuerzo?).

No todas las protestas ante las desigualdades se fundan en una promesa incumplida, los cambios estructurales van más allá de lo observable en los discursos. A la luz de los hechos, las demandas que promovieron y justificaron importantes revoluciones -la americana en 1776, la francesa en 1794, la mexicana 1910, la soviética en 1917 y la maoísta en 1949, entre otras-, no eliminaron el lado oscuro de las desigualdades. En la mayoría de los casos, se reemplazaron exclusiones escandalosas por otras que, en primera instancia, se consideraron más tolerables (según el caso: la libertad o la igualdad).

Las desigualdades que se relacionan con los procesos de inclusión/exclusión son consustanciales a la diferenciación funcional de la sociedad. No debería sorprender la multiplicación de las exclusiones, más debería llamar la atención la apertura a declaraciones que las tratan como problemas remediables, sin reconocer que son producidas por las propias operaciones de inclusión que se han abierto con la nueva estructura social. De hecho, los requerimientos de selección, de los cuales se hace cargo la educación, han aumentado en relación con el incremento de la complejidad de la sociedad, y en otro vértice, la participación política impulsada por la ampliación de la democracia ha dejado en evidencia a las minorías y sus reclamos por ser efectivamente considerados.

Un obstáculo que limita la comprensión de las nuevas formas de desigualdad social consiste en asociarlas mecánicamente con la estratificación económica intergeneracional. Especialmente cuando se constata que 'los mismos de siempre' ocupan las posiciones de privilegio gracias a sus redes familiares, no pagando impuestos, con leyes especiales, influyendo en las decisiones políticas o explotando, por 'razones' de clase o linaje, a quienes participan en la construcción de la riqueza. Es decir, con formas inaceptables. Pero es insostenible la expectativa de que, en última instancia, la exclusión sería solamente un problema de distribución de los ingresos o de la eliminación de la pobreza, y que de ello resultaría la inclusión global y duradera. No se tienen experiencias de eso.

La diferenciación funcional, como señalamos, no inhibe la producción de las desigualdades económicas. Sus efectos en ingresos precarios, falta de trabajo o bajas jubilaciones pueden ser devastadores; pero limitarse solo a ellas ignora exclusiones que refieren, por ejemplo a las orientaciones sexuales fuera de la norma, a la condición de trabajador extranjero, a la apariencia física, al estado civil, a las estadías en cárceles, al desempeño de oficios no valorados, a las minusvalías, al origen étnico, a la condición de madre soltera, a las enfermedades crónicas o a la soledad. De hecho, muchas de estas condiciones no pueden explicarse como efectos de las desigualdades económicas, pues más bien son las que las provocan. En otro sentido, tampoco la fortuna y los apellidos son garantía de salud, familias integradas y felices, sabiduría o seguridad personal. Sin contextualizar no es fácil definir las peores situaciones, aunque es evidente que sobre la pobreza material puede actuarse en forma más estándar y efectiva (de ahí el atractivo de concentrarse en ella). No obstante, en la sociedad contemporánea las demandas por inclusión no solo se han intensificado, sino que también se han diversificado, y los niveles de desigualdades relativas pueden ser más relevantes que los absolutos. 


\section{El rol estratégico (pero limitado) de la política}

Las demandas de mayor inclusión ejercen una permanente redefinición de los programas con los cuales se definen los límites entre la justicia y la injusticia y sus cruces con la igualdad y la desigualdad. Este tema remite a la política, ya que desde ella las exclusiones sociales pueden relativizarse y abrirse a considerar márgenes para su aceptabilidad. En este plano, como señaló Stacy Adams (1965), las diferencias percibidas como injustas constituyen una importante fuente de tensiones -'rabias'. El contenido de esa observación debe ser tomado en cuenta, pues los componentes sistémicos de la sociedad producen 'naturalmente' desigualdades sociales. La forma estructural de la sociedad imposibilita la inclusión total; más aun, dada su multidimensionalidad, y en una visión transversal, hace más probables las exclusiones. Estas últimas están en constante expansión, aunque sometidas a observaciones que las evalúan y las enfrentan con menor o mayor tolerancia, ya que no todas pesan lo mismo en lo que respecta a los balances de integración social y menos para sus distintos observadores.

Las protestas sociales impelen a los sistemas, especialmente, al político, a que se las tome en cuenta -para lo cual se acoplan con los medios de comunicación para las masas. Por ello, en todos los países modernos se diseñan políticas con el objeto de controlar o limitar las exclusiones, especialmente las más impopulares -las desigualdades en los ingresos- 0 aquellas que incrementan los gastos del erario público y desvían sus recursos. Pero, con respecto a las primeras, aunque se obtenga muy poco, las demandas se calman, al menos transitoriamente, cuando son atendidas bajo formas políticas.

Los esfuerzos político-estatales echan mano a los impuestos y a sus funciones redistributivas, buscan reparar aspectos que dicen relación con estándares mínimos de la existencia humana y, también, cuando las protestas disminuyen, actualizan los derechos exigibles, acompasándolos con nuevos conocimientos e ideologías en torno al bienestar disponible alcanzado en la sociedad.

Ciertamente, las presiones a la organización estatal arrancan de la expectativa de vigencia de un orden donde la política controlaba la sociedad. Como ello se cumple muy limitadamente, la decepción es generalizada, pero ello no paraliza las protestas, por el contrario, pueden, como está ocurriendo, apuntar a movilizaciones más masivas y desestabilizantes en donde la misma política y sus agentes se incluyen como el objeto de la protesta.

Las fórmulas organizativas más conocidas de la política moderna como los estados socialistas, el estado de bienestar y el estado subsidiario, reconocen las exclusiones como temporales y acotadas, ello les abre el camino para identificarlas, priorizarlas y orientar sus decisiones. En el intertanto, se diferencian por sus formas, activas o pasivas, de actuar frente a las estructuras tradicionales: nivelando forzadamente, con redes acotadas de protección, o esperando que el chorreo empareje las diferencias. Así, por ejemplo, aunque no hay evidencias de que los sistemas sociales se subordinen mecánicamente entre sí, se presume que mejores condiciones de empleo, niveles educativos más altos o el reforzamiento de la participación ciudadana estarían asociadas a condiciones que hacen más probables las inclusiones positivas (o negativas en su carencia), pues se supone que incrementan las posibilidades de coordinar sus ventajas y sacarles un mayor rendimiento.

En todo caso, los programas con que opera el código inclusión/ exclusión en cada uno de los sistemas sociales facilitan el que los planificadores, haciéndose eco del discurso público y de las necesidades de sus empleadores, propongan medidas, por ejemplo, indicar las condiciones que se asocian con el aumento de las posibilidades de acumulación de exclusiones, para evitar o para limitar interdependencias no deseadas (Nassehi 2000). De este modo se actúa frente al empleo precario, la baja cobertura o calidad educacional, la ausencia de redes familiares o sociales o una mala salud pública -como la falta de 
prevención ante las epidemias o las enfermedades de alto costo. Así la política, a través de sus organizaciones, recompone las situaciones más críticas desviando los déficits en direcciones más manejables, por ejemplo con internaciones, subsidios, reubicaciones, represión, bonos, etcétera.

Aunque no habría impedimentos para evitar algunas exclusiones, ello requiere mayores especificaciones (e.o. Thumala, Arnold \& Urquiza 2010). Pero se advierte que no es posible activar reparaciones sin provocar efectos. Por ejemplo, las políticas de becas desencadenan una competencia por las mismas y algunos no las obtienen; la construcción de represas hidroeléctricas incide en los niveles de empleo, pero afecta a las comunidades aledañas, y las acciones afirmativas excluyen a quienes solo aspiran a movilizarse por sus méritos. Más aun, debe atenderse que la integración social trata con situaciones dinámicas, por ejemplo: los niños necesitan a sus familias, luego a sus grupos de pares y relaciones de amistad, en la edad mediana el trabajo es fundamental, y en la vejez se agregan a las anteriores las instituciones de salud. Finalmente, también las organizaciones dedicadas a la asistencia y protección social son productoras de exclusión, partiendo por la definición de sus membrecías y después en la selección de sus grupos objetivos (Arnold 2008b).

\section{Conclusiones: La revolución esperada}

Hemos señalado que la modalidad de organización de la sociedad contemporánea, más que eliminar, multiplica y diversifica las desigualdades sociales. Por eso no pueden señalarse como anomalías corregibles con decisiones o instructivos morales. La exclusión social no puede excluirse bajo su actual modalidad estructural, solamente puede procesarse, como la escasez, los distintos intereses, las diferentes apreciaciones de la belleza, la búsqueda de la verdad, etcétera.

Actualmente para contener las protestas 0 , al menos, encauzar las demandas por mayor inclusión social, se redefinen los vínculos entre la justicia y la igualdad (o su contrario: entre la injusticia y la desigualdad) y se renuevan permanentemente sus contenidos. Definir estos ámbitos es, hasta ahora, competencia de la política, cuya estabilidad estructural dependerá de las capacidades disponibles para acoger los conflictos que tengan mayor presencia. Entre estos, identificar aquellos que se originan de las desigualdades que atentan contra el anhelo de que, si somos excluidos, al menos lo seamos 'justamente'.

En todo caso, más allá de la descripción de las operatorias de las desigualdades -que hemos intentado hacer- , la superación de sus efectos no deseados solo puede provenir de un cambio radical en la programación de la diferenciación de la sociedad, es decir, contando con una forma de sociedad que admita, como su característica basal, estructuras que no excluyan y que, por lo tanto, no incluyan. Mientras ello no ocurra, la sociedad seguirá (auto) confrontándose, hasta donde lo permitan sus condiciones, desvinculando las desigualdades que (la sociedad) produce fácticamente de lo que en ella (la sociedad) normativamente se rechaza. Por ahora, pueden esperarse protestas, en todas partes del planeta, cada vez más amplias, frecuentes y diversificadas exigiendo los grandes cambios sociales que se requieren (enunciados como cambios del 'modelo', cambios del 'sistema' o retomando el lema 'pidamos lo imposible'). Mientras la revolución esperada no se produzca, la sociedad contemporánea seguirá ofreciendo las mejores condiciones para operar bajo una intensa conflictividad social -'ajustes de cuentas'-, cuya cobertura mundial, a través de su reiterada exposición en los medios de comunicación para las masas, impedirá ignorarla.

Podemos concluir que hasta ahora, sin duda, y lamentablemente, la evolución de la sociedad no ha dado con la fórmula para excluir las exclusiones. Esta hipótesis puede no 
ser una buena noticia, pero conviene tenerla en cuenta para comprender la intensidad de la actual crisis mundial y estimar su futuro. Desde allí puede estar constituyéndose, también, un interesante desafío para las ciencias sociales que quieran ir más allá de la interpretación del mundo. RM

\section{Bibliografía}

Adams, J. (1965). Inequity in Social Exchange. Adv. Exp. Soc. Psychol., 62, 335- 343.

Arnold, M (2008a). La sociedad como sistema autopoiético: fundamentos del programa sociopoiético. En, La nueva teoría social en Hispanoamérica. Introducción a la teoría de sistemas constructivista (pp. 46-71). México D.F.: Universidad Autónoma del Estado de México.

Arnold, M. (2008b). Las organizaciones desde la teoría de los sistemas sociopoiéticos. Cinta de Moebio, 32.

Cadenas, H. (2012). La desigualdad de la sociedad. Diferenciación y desigualdad en la sociedad moderna. Persona y Sociedad, 26(2), 51- 77.

Lockwood, D. (1964). Social Integration and System Integration. En G. Zollschan \& W. Hirsch (Eds.), Explorations in Social Change (pp. 244- 257). Boston: Houghton Mifflin.

Luhmann, N. (1985). Zum begriff der sozialen Klasse. En N. Luhmann (Ed.), Soziale Differenzierung Zur Geschichte einer Idee (pp. 119-162). Opladen: Westdeutscher Verlag.

Luhmann, N. (1991). Die Form «Person». Soziale Welt, 42(2), 166- 175.

Luhmann, N. (1995). Inklusion und Exklusion. En Soziologische Aufklärung 6. Die Soziologie und der Mensch (pp. 237- 264). Opladen: Westdeutscher Verlag.

Luhmann, N. (1998). Die Gesellschaft der Gesellschaft. Frankfurt a.M.: Suhrkamp.

Mascareño, A. (2008). Acción, estructura y emergencia en la teoría sociológica. Revista de Sociología - Universidad de Chile, 22, 217- 256.

Mathieson, J. et al (2008). Social Exclusion. Meaning, Measurement and Experience and Links to Health Inequalities. A Review of Literature. WHO Social Exclusion Knowledge Network Backgropund Paper 1.

Nassehi, A. (2000). „Exklusion“ als soziologischer oder sozialpolitischer Begriff? Mittelweg, $36(5), 18-25$.

Robles, F. (2000). El desaliento inesperado de la modernidad. Molestias, irritaciones y frutos amargos de la sociedad del riesgo. Santiago de Chile: Ediciones Sociedad Hoy, RIL Editores.

Stichweh, R. (1997). Inklusion/Exklusion, funktionale Differenzierung und die Theorie der Weltgesellschaft. Soziale Systeme, 3(2), 123- 136.

Thumala, D., Arnold, M. \& Urquiza, A. (2010). Modalidades de inclusión/exclusión social de las personas adultas mayores: observaciones de la población chilena. Santiago de Chile: Universidad de Chile, SENAMA.

\section{Sobre el autor}

Marcelo Arnold Cathalifaud es actualmente Vicepresidente de la Asociación Latinoamericana de Sociología (ALAS) y Decano de la Facultad de Ciencias Sociales de la Universidad de Chile. Doctor en Ciencias Sociales de la Universidad de Bielefeld (Alemania). Antropólogo social y Magíster en ciencias sociales de la Universidad de Chile. Entre sus áreas de especialización se encuentran: teoría de sistemas sociales, programa sociopoiético, estudios organizacionales, problemas sociales emergentes, envejecimiento poblacional, exclusión social, solidaridad y colaboración social. Entre sus últimas publicaciones se destacan: En las vísperas de una revolución ignorada: el actual envejecimiento demográfico y sus consecuencias (en co-autoría con Daniela Thumala y Anahí Urquiza, 2011) y Constructivismo sociopoiético (2010).

marnold@uchile.cl 


\section{Contacto}

Universidad de Chile

Facultad de Ciencias Sociales

Av. Capitán Ignacio Carrera Pinto 1045,

Ñuñoa, Santiago de Chile

Chile

Recibido: Junio 2012

Aceptado: Agosto 2012 